\title{
Low compressibility accretion disc formation in close binaries: the role of physical viscosity
}

\author{
G. Lanzafame ${ }^{1}$, G. Belvedere ${ }^{2}$, and D. Molteni ${ }^{3}$ \\ 1 INAF - Osservatorio Astrofisico di Catania, via S. Sofia 78, 95123 Catania, Italy \\ e-mail: glanzafame@oact.inaf.it \\ 2 Dipartimento di Fisica e Astronomia dell'Universitá - Sezione Astrofisica, via S. Sofia 78, 95123 Catania, Italy \\ 3 Dipartimento di Fisica e Tecnologie Relative, Universitá di Palermo, Viale delle Scienze, 90128 Palermo, Italy
}

Received 19 September 2005 / Accepted 9 March 2006

\begin{abstract}
Aims. Physical viscosity naturally hampers gas dynamics (rarefaction or compression). Such a role should support accretion disc development inside the primary gravitation potential well in a close binary system, even for low compressibility modelling. Therefore, from the astrophysical point of view, highly viscous accretion discs could exist even in the low compressibility regime showing strong thermal differences to high compressibility ones

Methods. We performed simulations of stationary Smooth Particle Hydrodynamics (SPH) low compressibility accretion disc models for the same close binary system. Artificial viscosity operates in all models. The absence of physical viscosity and a supersonic high mass transfer characterize the first model. Physical viscosity and the same supersonic high mass transfer characterize the second model, whilst physical viscosity and a subsonic low mass transfer characterize the third model. The same binary system parameters, such as stellar masses and their separation, have been adopted, as well as the same polytropic index $\gamma=5 / 3$. Thus we investigated the role of physical viscosity in mass and angular momentum transport in the two viscid models and compare them to the inviscid model. An initial value of the parameter $\alpha=1$ has been considered for the physically viscous models, according to the well-known Shakura and Sunjaev formulation, but simulations were carried out also for $\alpha=0.1$ and $\alpha=0.5$ in the case of a supersonic mass transfer. Physical viscosity is represented by the viscous force contribution expressed by the divergence of the symmetric viscous stress tensor in the Navier-Stokes equation, while the viscous energy contribution is given by a symmetric combination of the symmetric shear tensor times the particle velocity.

Results. The results show that physical viscosity supports and favours accretion disc formation despite the very low compressibility assumed. On the contrary, in the inviscid case no evident disc structure appears. In all models neither shock fronts nor extended clear spirals in the radial flow develop.
\end{abstract}

Key words. accretion, accretion discs - stars: binaries: close - stars: dwarf novae, white dwarfs - methods: numerical methods: N-body simulations

\section{Introduction}

Since the eighties, the existence of spiral patterns and radial shocks in physically inviscid accretion discs in close binaries has been discussed. According to some authors, i.e. Chakrabarti (1992), Bisikalo et al. (1999), spiral patterns do not appear if a polytropic index (appearing in the perfect gas state equation) $\gamma>1.16$ is adopted. According to some others, such as Sawada et al. (1987), Spruit et al. (1987), Kaisig (1989), Matsuda et al. (1990, 1992), Ichikawa \& Osaki (1992, 1994), Sawada \& Matsuda (1992), Savonije et al. (1994), Yukawa et al. (1997), Makita et al. (2000), spiral patterns and radial shocks always exist whatever the $\gamma$ is and the initial kinematic conditions are. Tidal effects should be responsible for spirals and spiral shock development in the disc bulk, starting from the outer disc edge. Recently some papers (Lanzafame et al. 2000, 2001; Lanzafame \& Belvedere 2001; Belvedere \& Lanzafame 2002; Lanzafame \& Belvedere 2005) conclude, through 2D and 3D SPH simulations, that initial conditions, such as angular momentum and energy, are crucial in spiral shock formation. These studies were performed as a consequence of the fact that in some previous papers of our group (Molteni et al. 1991;
Lanzafame et al. 1992) we did not obtain spirals, both in low compressibility models $(\gamma=1.1$ and $\gamma=1.2)$ and in a high compressibility model $(\gamma=1.01)$. Instead, we found spiral patterns, mainly close to the disc outer edge, in Lanzafame \& Belvedere (1997, 1998) by considering different injection conditions. In Molteni et al. (1991) and in Lanzafame et al. (1992) no well bound and defined accretion discs were found in the case of low compressibility models $(\gamma=1.1$ and $\gamma=1.2)$. The investigation carried out in this paper arose as a consequence of the idea that physical viscosity naturally hampers the gas dynamics (rarefaction or compression). Such a role should support accretion disc development inside the primary gravitation potential well, even for low compressibility modelling. In the case of a well bound and defined physically viscous accretion disc, we also investigated the possibility of spiral shock front development from its outer edge in spite of the physical viscosity degradation effect on gas dynamics and shock front discontinuities. Spiral shock fronts coming out in the disc radial flow have clearly been shown in a previous paper (Lanzafame 2003) for $\gamma=1.01$ in the case of a physically viscous disc. In this paper a $\gamma=5 / 3$ polytropic index has been adopted and a comparison among three stationary SPH accretion disc models has been performed, adopting 
the same binary system parameters such as stellar masses and their separation. The here adopted $\gamma=5 / 3$ value is common in the literature. Even higher $\gamma$ s up to 2 have been chosen by various authors (e.g. Bisikalo et al. 1999). However in most papers the gas chemical composition is unknown or not specified. This means that the $\gamma$ value does not have any strict relation to the traditional adiabatic index built using specific heats.

In this paper the first model (model a)) is of a physically inviscid accretion disc and adopts a supersonic high mass transfer condition at the inner Lagrangian point L1. The second one (model b)) is a physically viscous disc with the same supersonic mass trasfer condition at L1. The third one (model c)) is a physically viscous disc and adopts a subsonic low mass transfer condition at L1. The well-known Shakura and Sunyaev viscosity prescription (Shakura 1972; Shakura \& Sunyaev 1973) has been adopted with $\alpha=1$.

The SPH formulation of viscous contributions in the NavierStokes and energy equations has been developed by Flebbe et al. $(1992,1994)$. Such a formulation could also describe molecular and turbulent viscosity effects (Landau \& Lifshitz 1959). These goals are not obtained by artificial viscosity which is, however, introduced in both models to resolve shocks numerically and to avoid spurious heating. Artificial viscosity vanishes when the limit value of the particle interpolation domain goes to zero. Drimmel (1993), Meglicki et al. (1993), Murray (1996) demonstrated that the linear component of artificial viscosity itself, in the continuum limit, yields a viscous shear force. In particular the last two authors have explicitly formulated such an artificial viscosity contribution in the momentum and energy equations. Murray (1996) found an analogy between the shear viscosity generated by the linear artificial viscosity term and the well-known Shakura and Sunyaev shear viscosity, in the continuum limit. These conclusions do not disagree with our goals. The SPH method, like other finite difference schemes, is far from the continuum limit; moreover we need the quadratic $(\beta)$ artificial viscosity term to handle strong shocks. In conclusion, we compare three SPH accretion disc models where artificial viscosity is necessarily included. Some models also include physical viscosity, but one does not. In this paper, the viscous force contribution is represented by the divergence of the symmetric viscous stress tensor in the Navier-Stokes equation. A symmetric combination of the symmetric shear tensor times the particle velocity has been added to the energy equation as a viscous heating contribution. The bulk physical viscosity contribution has not been considered for the sake of simplicity.

Notice that our analysis and results are somewhat different from those by Flebbe et al. (1992, 1994), who used the same $\mathrm{SPH}$ viscous formulation in the equations and applied the code to similar numerical tests. However, they adopted a shear component of the stress tensor proportional to the $\alpha$ Shakura and Sunjaev parameter times the gas pressure and excluded any artificial viscosity contribution. Bisikalo et al. (2000), adopting $\gamma=1.01$, an Eulerian finite difference method and various physical viscosities, found self-consistent solutions showing the absence of a shock impact between the stream flowing from L1 and the disk body at its outer edge. They also found a similarity of the flow patterns even adopting different disc viscosities.

In Sect. 2 of this paper we briefly present the SPH formulation of the models. In Sect. 3 we describe the adopted boundary conditions and the physical characteristics of the disc gas, while in Sect. 4 we discuss our results.

\section{SPH formulation of physically of both inviscid and viscous perfect gas hydrodynamics}

As for non-viscous gas hydrodynamics, the relevant equations to our model are:

$\frac{\mathrm{d} \rho}{\mathrm{d} t}+\rho \nabla \cdot \underline{v}=0$

continuity equation

$\frac{\mathrm{d} \underline{v}}{\mathrm{~d} t}=-\frac{\nabla p}{\rho}+\left[-2 \underline{\omega} \times \underline{v}+\underline{\omega} \times(\underline{\omega} \times \underline{r})-\nabla \Phi_{\text {grav }}\right]$

momentum equation

$\frac{\mathrm{d} \epsilon}{\mathrm{d} t}=-\frac{p}{\rho} \nabla \cdot \underline{v}$

energy equation

$p=(\gamma-1) \rho \epsilon$

perfect gas equation.

Here most symbols have the usual meaning, $\mathrm{d} / \mathrm{d} t$ stands for the Lagrangian derivative, $\rho$ is the gas density, $\epsilon$ is the thermal energy per unit mass, $\Phi_{\text {grav }}$ is the effective gravitational potential generated by the two stars and $\underline{\omega}$ is the angular velocity of the rotating reference frame, corresponding to the rotational period of the binary system. Self gravitation has not been included, as it appears irrelevant. The adiabatic index $\gamma$ is a numerical parameter whose value lies in the range between 1 and $5 / 3$, in principle.

The SPH method is a Lagrangian scheme that discretizes the fluid into moving interacting and interpolating domains called "particles". All particles move according to pressure and body forces. The method makes use of a kernel $W$ useful to interpolate a physical quantity $A(\underline{r})$ related to a gas particle at position $\underline{r}$ according to:

$A(\underline{r})=\int_{D} A\left(\underline{r}^{\prime}\right) W\left(\underline{r}, \underline{r}^{\prime}, h\right) \mathrm{d} \underline{r}^{\prime}$

$W\left(\underline{r}, \underline{r}^{\prime}, h\right)$, the interpolation kernel, is a continuous function or two connecting continuous functions whose derivatives are continuous even at the connecting point - defined in the spatial range $2 h$, whose limit for $h \rightarrow 0$ is the Dirac delta distribution function. All physical quantities are described as extensive properties smoothly distributed in space and computed by interpolation at $\underline{r}$. In SPH terms we write:

$A_{i}=\sum_{j=1}^{N} \frac{A_{j}}{n_{j}} W\left(\underline{r}_{i}, \underline{r}_{j}, h\right)$

where the sum is extended to all particles included in the domain $D, n_{j}=\rho_{j} / m_{j}$ is the number density relative to the $j$ th particle and $W\left(\underline{r}_{i}, \underline{r}_{j}, h\right) \leq 1$ is the adopted interpolation kernel whose value is determined by the relative distance between particles $i$ and $j$.

In the SPH formalism, Eqs. (2) and (3) take the form:

$\frac{\mathrm{d} \underline{v}_{i}}{\mathrm{~d} t}=-\sum_{j=1}^{N} m_{j}\left(\frac{p_{i}}{\rho_{i}^{2}}+\frac{p_{j}}{\rho_{j}^{2}}\right) \nabla_{i} W_{i j}+\underline{g}_{i}$

$\frac{\mathrm{d} \epsilon_{i}}{\mathrm{~d} t}=\frac{1}{2} \sum_{j=1}^{N} m_{j}\left(\frac{p_{i}}{\rho_{i}^{2}}+\frac{p_{j}}{\rho_{j}^{2}}\right) \underline{v}_{i j} \cdot \nabla_{i} W_{i j}$ 
where $\underline{g}_{i}=-2 \underline{\omega} \times \underline{v}_{i}+\underline{\omega} \times\left(\underline{\omega} \times \underline{r}_{i}\right)-\nabla \Phi_{\text {grav }, i}, \underline{v}_{i j}=\underline{v}_{i}-\underline{v}_{j}$ and $m_{j}$ is the mass of the $j$ th particle. Alternatively, Eq. (8) can also be written:

$\frac{\mathrm{d} \epsilon_{i}}{\mathrm{~d} t}=\sum_{j=1}^{N} m_{j} \frac{p_{i}}{\rho_{i}^{2}} \underline{v}_{i j} \cdot \nabla_{i} W_{i j}$.

In this scheme the continuity equation takes the form:

$\frac{\mathrm{d} \rho_{i}}{\mathrm{~d} t}=-\sum_{j=1}^{N} m_{j} \underline{v}_{i j} \cdot \nabla_{i} W_{i j}$

or it can also be written as:

$\rho_{i}=\sum_{j=1}^{N} m_{j} W_{i j}$

which identifies the natural space interpolation of particle densities according to Eq. (6).

The pressure term includes the artificial viscosity contribution given by (Monaghan 1985, 1992) and Monaghan \& Lattanzio (1985), with an appropriate thermal diffusion term which reduces shock fluctuations. It is given by:

$\eta_{i j}=\alpha \mu_{i j}+\beta \mu_{i j}^{2}$

where

$\mu_{i j}=\frac{2 h \underline{v}_{i j} \cdot \underline{r}_{i j}}{\left(c_{\mathrm{s} i}+c_{\mathrm{s} j}\right)\left(r_{i j}^{2}+\xi\right)}$

with $c_{\mathrm{s} i}$ being the sound speed of the $i$ th particle, $\underline{r}_{i j}=\underline{r}_{i}-\underline{r}_{j}$, $\xi^{2} \ll h^{2}, \alpha \approx 1$ and $\beta \approx 2$. These $\alpha$ and $\beta$ parameters of the order of unity are usually adopted to damp oscillations past high Mach number shock fronts developed by non-linear instabilities (Boris \& Book 1973). These $\alpha$ and $\beta$ values were also adopted by Lattanzio et al. (1986). Lower $\alpha$ and $\beta$ values, as adopted by Meglicki et al. (1993), would develop more turbulence in the disc and possibly only one shock front at the impact zone between the infalling particle stream and the returning particle stream at the disc outer edge. In the physically inviscid SPH gas dynamics, angular momentum transport is mainly due to the artificial viscosity included in the pressure terms as:

$\frac{p_{i}}{\rho_{i}^{2}}+\frac{p_{j}}{\rho_{j}^{2}}=\left(\frac{p_{i}^{(G)}}{\rho_{i}^{2}}+\frac{p_{j}^{(G)}}{\rho_{j}^{2}}\right)\left(1+\eta_{i j}\right)$

where $p^{(G)}$ is the intrinsic gas pressure.

In the Lagrangian SPH modelling of physically viscous gas dynamics, we have to add the viscous force term to the right side of momentum Eq. (2). The resulting Navier-Stokes equation is:

$\frac{\mathrm{d} \underline{v}}{\mathrm{~d} t}=-\frac{\nabla p}{\rho}+\left[-2 \underline{\omega} \times \underline{v}+\underline{\omega} \times(\underline{\omega} \times \underline{r})-\nabla \Phi_{\text {grav }}\right]+\frac{1}{\rho} \nabla \cdot t$.

Here, the viscous stress tensor $t_{\alpha \beta}$ includes the positive first and second viscosity coefficients $\eta_{v}$ and $\zeta_{v}$ which are velocity independent and describe shear and tangential viscosity stresses $\left(\eta_{v}\right)$, and compressibility stresses $\left(\zeta_{v}\right)$ :

$t_{\alpha \beta}=\eta_{v} \sigma_{\alpha \beta}+\zeta_{v} \nabla \cdot \underline{v}$

where the shear

$\sigma_{\alpha \beta}=\frac{\partial v_{\alpha}}{\partial x_{\beta}}+\frac{\partial v_{\beta}}{\partial x_{\alpha}}-\frac{2}{3} \delta_{\alpha \beta} \nabla \cdot \underline{v}$.
In these equations the indexes $\alpha$ and $\beta$ are spatial indexes while tensors are written in bold characters. For simplicity we assume $\zeta_{v}=0$, however our code also allows different choices. As in Flebbe et al. (1992, 1994), defining

$V_{i \alpha \beta}=\sum_{j=1}^{N} \frac{m_{j-j i \alpha}}{\rho_{j}} \frac{\partial W_{i j}}{\partial x_{\beta}}$

as the SPH formulation of $\partial v_{\alpha} / \partial x_{\beta}$, the SPH equivalent of the shear is:

$\sigma_{i \alpha \beta}=V_{i \alpha \beta}+V_{i \beta \alpha}-\frac{2}{3} \delta_{\alpha \beta} V_{i \gamma \gamma}$

therefore, the SPH momentum equation equivalent is:

$$
\begin{aligned}
\frac{\mathrm{d} \underline{v}_{i}}{\mathrm{~d} t}= & -\sum_{j=1}^{N} m_{j}\left(\frac{p_{i}}{\rho_{i}^{2}}+\frac{p_{j}}{\rho_{j}^{2}}\right) \nabla_{i} W_{i j}+\underline{g}_{i} \\
& +\sum_{j=1}^{N} m_{j}\left(\frac{\eta_{v i} \sigma_{i}}{\rho_{i}^{2}}+\frac{\eta_{v j} \sigma_{j}}{\rho_{j}^{2}}\right) \cdot \nabla_{i} W_{i j}
\end{aligned}
$$

and the energy balance equation for the total energy $E=\epsilon+$ $\frac{1}{2} v^{2}$ is:

$\left.\frac{\mathrm{d}}{\mathrm{d} t}\left(\epsilon+\frac{1}{2} v^{2}\right)=-\frac{1}{\rho}(p \nabla \cdot \underline{v}-\nabla \cdot \underline{v} \cdot \boldsymbol{t})\right)$

which has the SPH equivalent:

$$
\begin{aligned}
\frac{\mathrm{d}}{\mathrm{d} t} E_{i}= & -\sum_{j=1}^{N} m_{j}\left(\frac{p_{i} \underline{v}_{i}}{\rho_{i}^{2}}+\frac{p_{j} \underline{v}_{j}}{\rho_{j}^{2}}\right) \cdot \nabla_{i} W_{i j} \\
& +\sum_{j=1}^{N} m_{j}\left(\eta_{v i} \frac{\boldsymbol{\sigma}_{i} \cdot \underline{v}_{i}}{\rho_{i}^{2}}+\eta_{v j} \frac{\boldsymbol{\sigma}_{j} \cdot \underline{v}_{j}}{\rho_{j}^{2}}\right) \cdot \nabla_{i} W_{i j}
\end{aligned}
$$

where $E_{i}=\left(\epsilon_{i}+\frac{1}{2} v_{i}^{2}\right)$.

A full justification of this SPH formalism can be found in Flebbe et al. $(1992,1994)$.

\section{Parameters and boundary conditions}

The characteristics of the binary system are determined by the masses of the two companion stars and their separation. We chose to model a system in which the mass $M_{1}$ of the primary compact star and the mass $M_{2}$ of the secondary normal star are equal to one $M_{\odot}$ and the distance between their centres is $d_{12}=10^{6} \mathrm{~km}$. The injection gas velocity at L1 is fixed to $v_{\text {inj }} \simeq 130 \mathrm{~km} \mathrm{~s}^{-1}$ for our inviscid first model (a)) and our viscous second model (b)) and to $v_{\text {inj }} \simeq 13 \mathrm{~km} \mathrm{~s}^{-1}$ as for the viscous third model (c)) while the injection gas temperature at L1 is fixed to $T_{\mathrm{o}}=10^{4} \mathrm{~K}$. Such a temperature value is slightly higher than the value corresponding to a normal $1 M_{\odot}$ star because, even as a first approximation, the radiative heating of the secondary surface due to lightening of the disc is taken into account. Gas compressibility is fixed by the adiabatic index $\gamma$ of $5 / 3$. In models a) and b) we chose a highly supersonic injection velocity at L1, to verify whether, by adopting a high angular momentum injection condition, spiral structures and shocks in the radial flow would appear in low compressibility 3D modelling, as shown by Lanzafame et al. $(2000,2001)$ in 2D, and by Lanzafame (2003) in 3D, high compressibility models. We and other authors (Meglicki et al. 1993; Yukawa et al. 1997) 
have found that spirals and shock fronts in the radial flow do not develop in high compressibility 3D SPH accretion disc models if subsonic injection conditions at L1 are adopted, in spite of the fact that tidally induced spiral patterns should develop (Murray 1996; Blondin 2000; Truss et al. 2000, 2001), at least in $2 \mathrm{D}$ modelling. Therefore, our assumption of strongly supersonic mass inflow could be effective enough to allow spirals to form in spite of the artificial viscosity degradation. In our models the unknowns are: pressure, density, temperature, velocity, so we solve the continuity, momentum, energy and state (perfect gas) equations.

In order to make our equations dimensionless, we adopted the following normalization factors: $M=M_{1}+M_{2}$ for masses, $d_{12}=10^{11} \mathrm{~cm}$ for lengths, $v_{\mathrm{o}}=\sqrt{G\left(M_{1}+M_{2}\right) / d_{12}}$ for speeds, so that the orbital period is normalized to $2 \pi, \rho_{\mathrm{o}}=10^{-9} \mathrm{~g} \mathrm{~cm}^{-3}$ for the density, $p_{\mathrm{o}}=\rho_{\mathrm{o}} v_{\mathrm{o}}^{2}$ dyn $\mathrm{cm}^{-2}$ for pressure, $v_{\mathrm{o}}^{2}$ for thermal energy per unit mass and $T_{\mathrm{o}}=(\gamma-1) v_{\mathrm{o}}^{2} m_{\mathrm{p}} K_{\mathrm{B}}^{-1}$ for temperature, where $m_{\mathrm{p}}$ is the proton mass and $K_{\mathrm{B}}$ is the Boltzman constant. The adopted kernel width in the SPH interpolations is $h=0.005$ and the geometrical domain including moving disc particles is a sphere of radius 0.6 , centred on the primary. The rotating reference frame is centred on the compact primary and its rotational period equals the orbital one. We simulated the physical conditions at the inner and at the outer edges as follows:

a) Inner edge:

The free inflow condition is realized by eliminating particles flowing inside the sphere of radius $4 h$, centred on the primary. It follows that disc structure and dynamics are altered within a few $h$ values of the inner edge, adopting a fixed $h$ SPH code, but these alterations in disc behaviour are relatively small because they are counterbalanced by a higher particle concentration close to the inner edge in the supersonic injection models.

b) Outer edge:

The injection of "new" particles from L1 towards the interior of the primary Roche Lobe is simulated by generating them in fixed points, called "injectors", symmetrically placed within an angle having $\mathrm{L} 1$ as a vertex and an aperture of $\sim 57^{\circ}$. The initial injection particle velocity is radial with respect to L1. In order to simulate a constant and smooth gas injection, a "new" particle is generated in the injectors whenever "old" particles leave an injector free, inside a small sphere with radius $h$, centred on the injector itself.

The formulation adopted for the 3D SPH accretion disc model including physical viscosity is the well-known Shakura (1972); Shakura \& Sunyaev (1973) $\alpha$ parametrization: $v=$ $\alpha c_{\mathrm{s}} H$, where $c_{\mathrm{s}}$ is the sound velocity, $0 \leq \alpha \leq 1$ is the Shakura and Sunyaev parameter and $H=r_{x y} c_{\mathrm{s}} / \sqrt{M_{1} / r_{x y}}$ is a dimensionless estimate of the standard disc thickness, where $r_{x y}=\sqrt{X_{i}^{2}+Y_{i}^{2}}$ is the cylindrical radial coordinate of the $i$ th particle. In this paper we chose to adopt $\alpha=1$ as an initial value to point out evident differences in disc structure and dynamics between our SPH disc models.

\section{Results and discussion}

\subsection{General results}

We carried out our simulations until a time $t=25 \simeq 3.98$ orbital periods, namely until fully stationary configurations. This means that particles injected into the primary potential well (which is not deep, due to the primary small mass) are statistically balanced by particles accreted onto the primary itself and by particles ejected from the outer disc edge. In the present simulations,

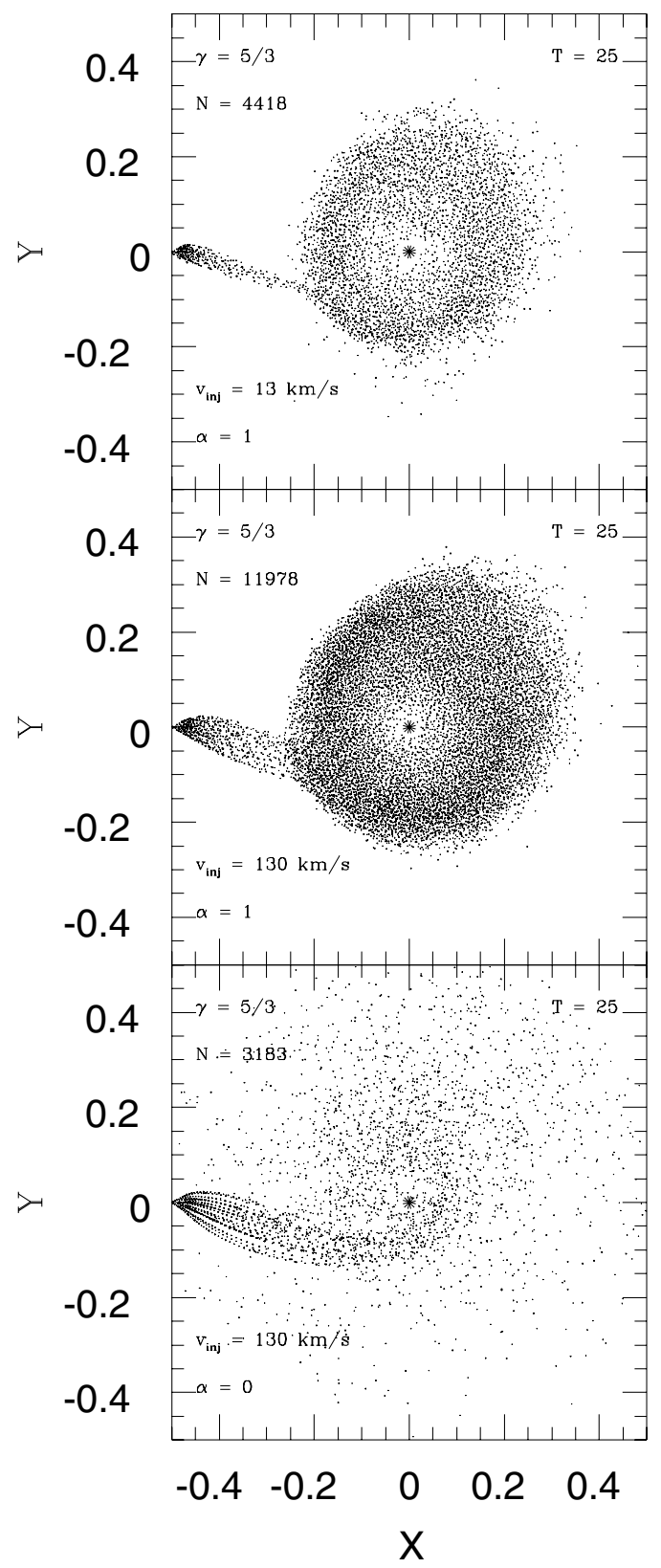

Fig. 1. $X Y$ plots for the inviscid model a) with $\alpha=0$ (bottom) and the two physically viscous models with $\alpha=1$ (middle - model b) and topmodel c)). The final time $T$ and total particle number $N$, as well as the injection velocity from the inner Lagrangian point L1, are also reported.

in the fully stationary statistical equilibrium configurations, particles number only a few thousands ranging from about 3000 to about 13000 . There is no way to obtain a higher number of particles with the adopted value of $h=0.005$, which is a small enough value and historically confirmed since our first papers on accretion discs in 1991. Other authors have adopted such a good compromise $h$ value in SPH accretion disc modelling. However, here the problem is not the total number of particles, but rather their concentration, namely whether the fluid properties (density, pressure gradients, etc.) are computed accurately enough. This will be extensively discussed below (Sect. 4.2).

The mass transfer injection rates from L1 $\dot{M}_{\text {inj }}$, accretion rates $\dot{M}_{\text {acc }}$ and ejection rates $\dot{M}_{\text {eje }}$ are of the order of $\dot{M}_{\text {inj }} \sim$ $10^{17} \mathrm{~g} \mathrm{~s}^{-1}, \dot{M}_{\text {acc }} \sim 4.5 \times 10^{16} \mathrm{~g} \mathrm{~s}^{-1}$ and $\dot{M}_{\text {eje }} \sim 5.5 \times 10^{16} \mathrm{~g} \mathrm{~s}^{-1}$ for 


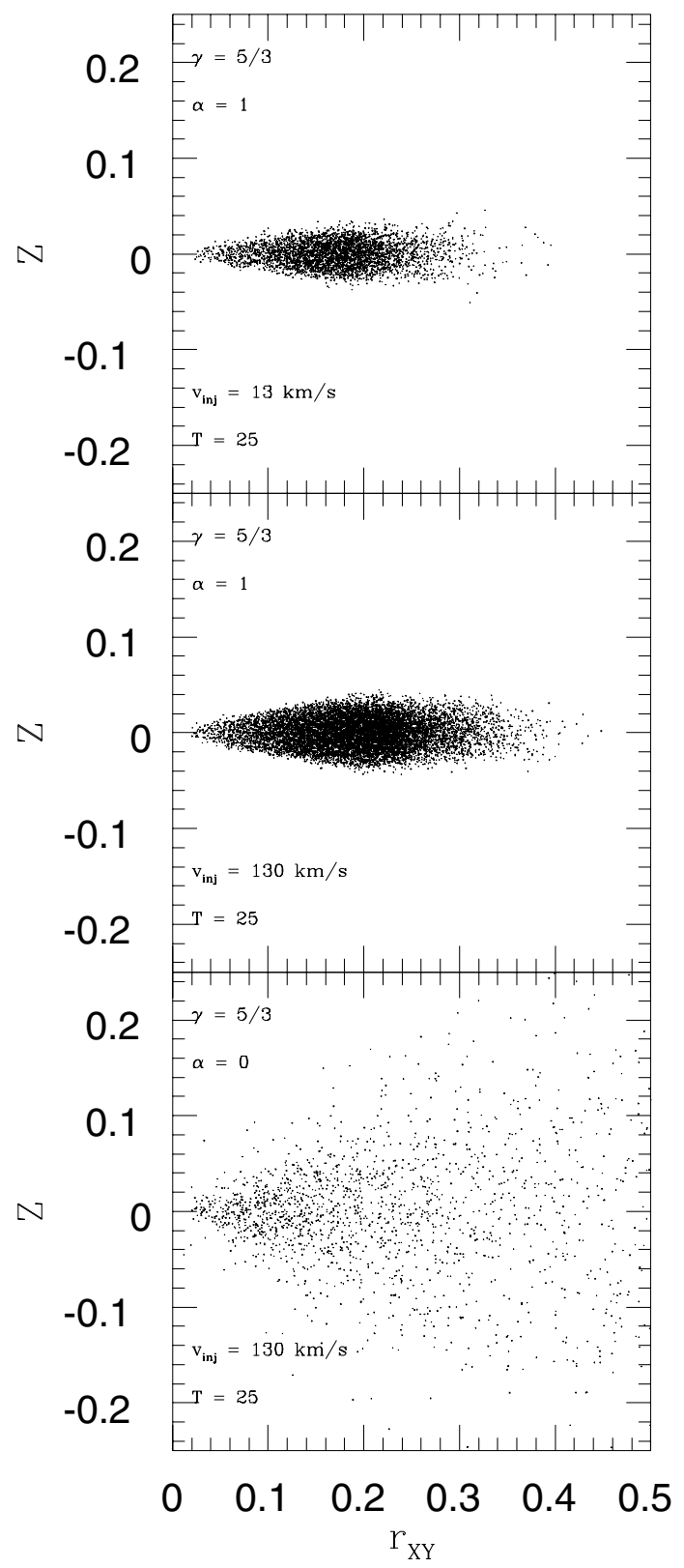

Fig. 2. $r_{X Y} Z$ plots for the inviscid model a) with $\alpha=0$ (bottom) and the two physically viscous models with $\alpha=1$ (middle - model b) and top model c)). The final time $T$ and total particle number $N$, as well as the injection velocity from the inner Lagrangian point L1, are also reported.

inviscid model a), $\dot{M}_{\text {inj }} \sim 6.5 \times 10^{16} \mathrm{~g} \mathrm{~s}^{-1}, \dot{M}_{\text {acc }} \sim 6.5 \times 10^{16} \mathrm{~g} \mathrm{~s}^{-1}$ and $\dot{M}_{\text {eje }} \sim 6.5 \times 10^{13} \mathrm{~g} \mathrm{~s}^{-1}$ for viscous disc model b) and $\dot{M}_{\text {inj }} \sim$ $2.0 \times 10^{16} \mathrm{~g} \mathrm{~s}^{-1}, \dot{M}_{\text {acc }} \sim 2.0 \times 10^{16} \mathrm{~g} \mathrm{~s}^{-1}$ and $\dot{M}_{\text {eje }} \sim 6.5 \times 10^{13} \mathrm{~g} \mathrm{~s}^{-1}$ for viscous disc model c). Both physically viscous disc models (b) and c)) show mass ejection rates lower than or equal to $\rho_{\mathrm{o}} h^{3} d_{12}^{2} v_{\mathrm{o}}$, which is the conversion factor from particle/time to $\mathrm{g} \mathrm{s}^{-1}$. For this reason we believe that the two viscous mass ejection rates may be considered as higher limits.

Figure 1 shows $X Y$ and $X Z$ plots of the physically inviscid disc (bottom-left side) and the two physically viscous discs (top side). While the latter plots clearly show well bound structures with a definite disc outer edge, the first plot shows that the particle dispersion is too high as to get a well bound and a well defined disc. This result was obtained by

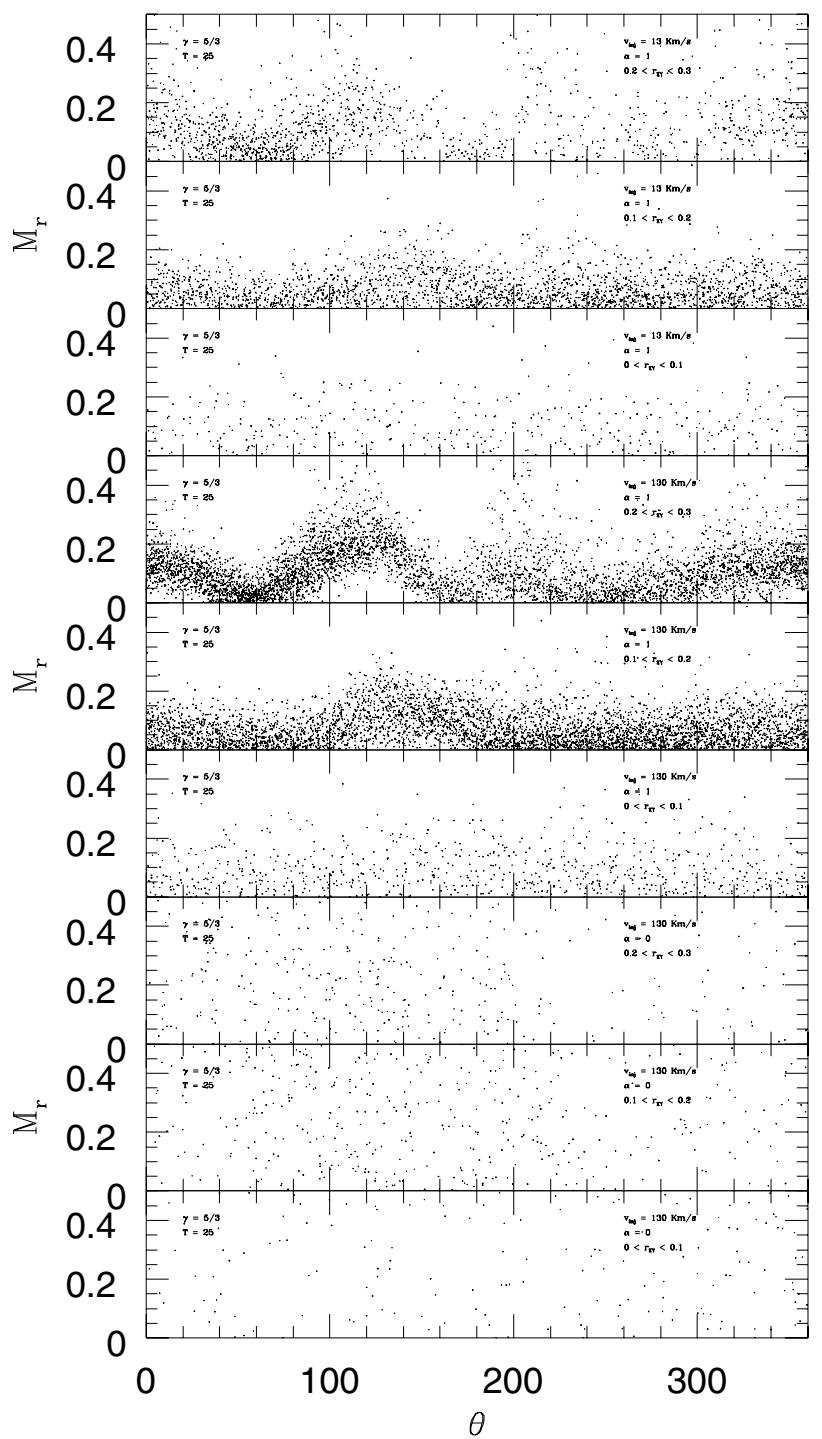

Fig. 3. Plots of the radial Mach number $M_{r}$ as a function of the radial distance for the inviscid model a) $(\alpha=0)$ and for the two physically viscous models: model b) for $\alpha=1, v_{\text {inj }}=130 \mathrm{~km} \mathrm{~s}^{-1}$ and model c) for $\alpha=1, v_{\text {inj }}=13 \mathrm{~km} \mathrm{~s}^{-1}$. Here three radial shells have been considered. The thickness of each radial shell is $\Delta r_{X Y}=0.1$, as shown in each plot by looking at $r_{X Y}$.

Molteni et al. (1991); Lanzafame et al. (1992), adopting lower polytropic indexes $\gamma=1.1$ and $\gamma=1.2$ and a subsonic injection condition at L1. Here we adopt the value $\gamma=5 / 3$ which represents the correct adiabatic index of a monoatomic gas.

Figure 2 shows the $r_{X Y} Z$ for all three accretion disc models obtained by folding all disc bulk particles onto a plane containing the $Z$ axis and being perpendicular to the $X Y$ orbital plane. An evident latitudinal spread appears for the inviscid model a), differently from the viscous models b) and c). Moreover, comparing models b) and c) shows a small but clear dependence on the mass transfer injection speed at L1. Latitudinal angular spreads computed for all three disc models are $\simeq 60^{\circ}, \simeq 24^{\circ}$ and $\simeq 20^{\circ}$ for disc models a), b) and c) respectively. Therefore, a more stretched and slim structure characterizes physically viscous discs due to the more effective particle radial velocity transport and channeling towards the primary.

Physical viscosity naturally works where the particle mutual velocity (and separation) change in time, namely when a mutual acceleration exists contrasting gas dynamics (rarefaction 


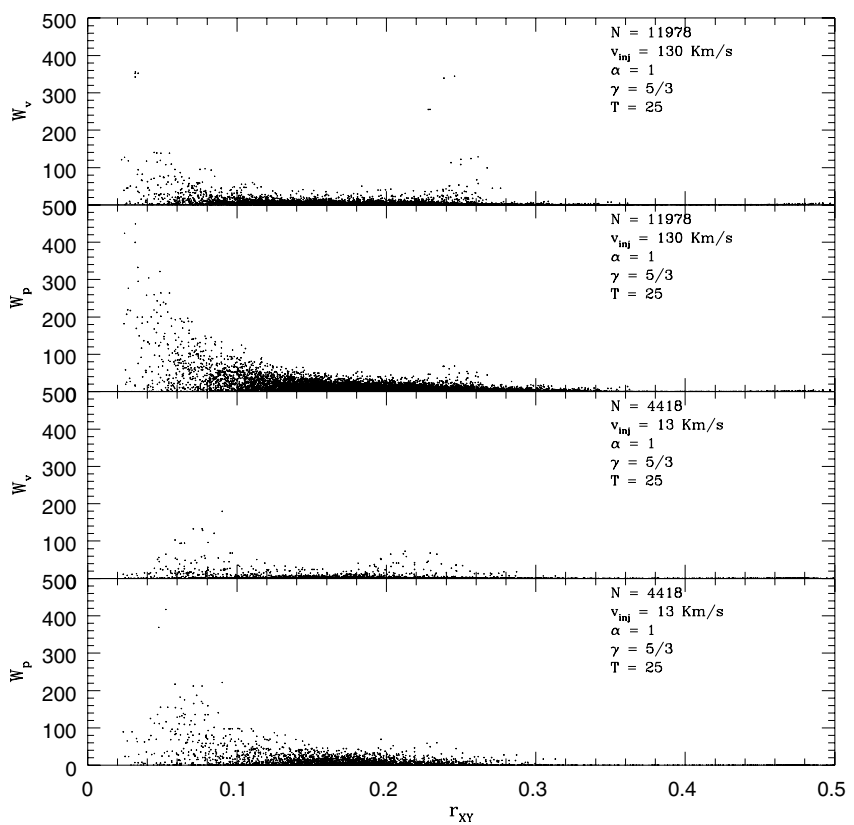

Fig. 4. Plots of the work made by the pressure forces $W_{p}$ and by the viscosity forces $W_{v}$ per unit mass (here $(\nabla p / \rho$ and $\left.\nabla \cdot \boldsymbol{t}) / \rho\right)$ ) as a function of the disc radial distance for model b) - (top two panels) and model c) - (bottom two panels).

or compression) and converting kinetic energy into thermal energy. Such a mechanism clearly supports the development of well bound accretion discs inside the primary potential well, in spite of the low compressibility, at least for $\alpha=1$. No spirals and shock fronts exist in the inviscid accretion disc bulk or at its outer edge, as shown in Figs. 1 and 2. The mean particle concentration is too low to allow us to have a reliable particle statistics for such an inviscid model. Because of this statistical limitation, we should be able to see spirals in a better defined physically viscous disc structure and especially in accretion disc model b) where a supersonic mass transfer injection condition has been adopted at L1, but it is not so. Figure 3 shows the profile of the radial Mach number $M_{r}$ as a function of the radial distance for all three accretion disc models. For a better understanding of such behaviour, a selection of particles included in three suitable cylindrical radial shells has been made for each model. Figure 3 shows that no shock fronts and spiral structures develop in all three accretion disc models. This is not surprising for models b) and c). Indeed, physical viscosity, slowing down the radial flow and heating the gas particles (so increasing the local sound speed), reduces the Mach number and the radial Mach number, although a more ordered radial transport, and some radial and azimuthal ripples, are clearly shown. However, we cannot conclude that spirals and shock fronts should not appear in the inviscid accretion disc since model a) is not well resolved because of the lack of reliable particle statistics. The lack of resolution is due not only to the low particle concentration but also to the artificial viscosity smoothing effect which is heavy in 3D simulations. This investigation shows that spirals and shock fronts may not develop in low compressibility physically viscous discs in a binary system with low mass stars and a mass ratio of the order of the unity. Our work is essentially concerned with viscid discs, the inviscid case being of minor importance. This is also the reason why 2D SPH simulations (where artificial viscosity is less important) are not totally overcome. On the contrary, often 2D simulations are complementary.

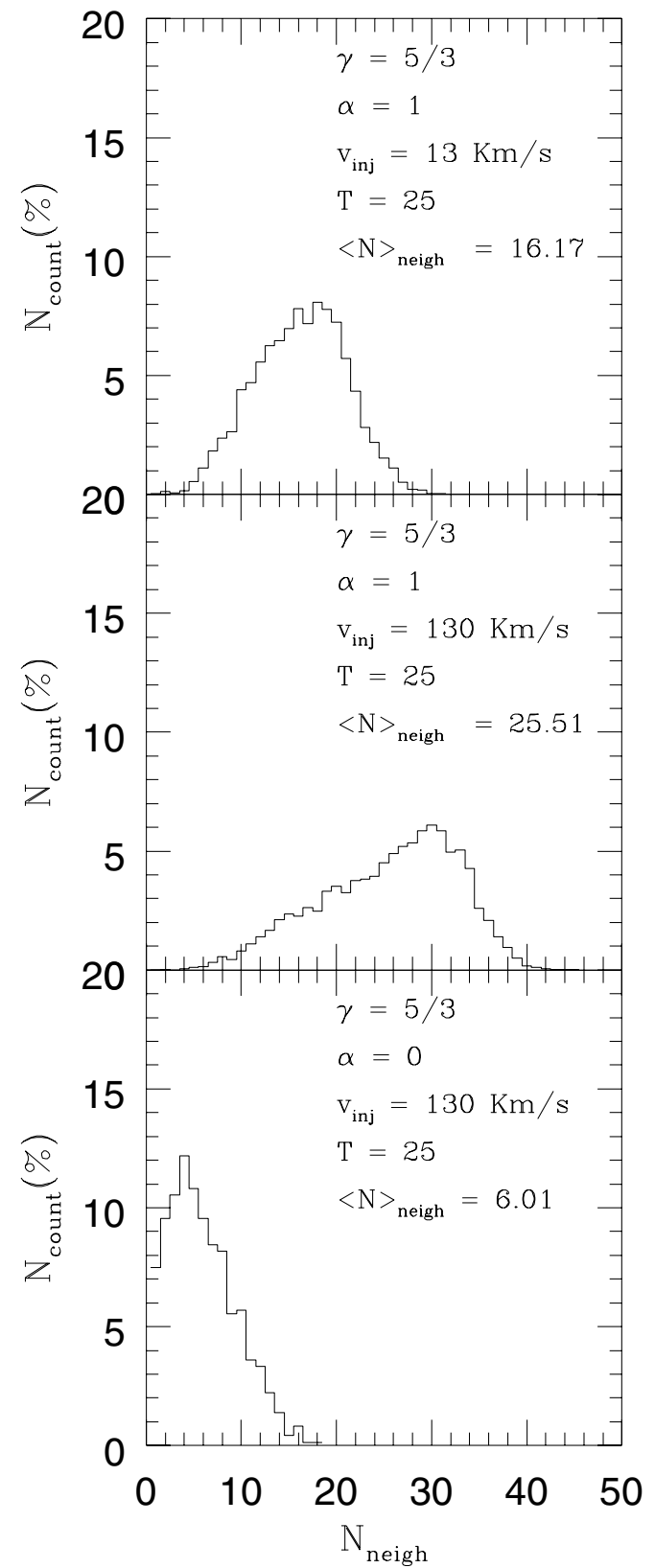

Fig. 5. Neighbours histograms for the inviscid model a) with $\alpha=0$ (bottom) and the two physically viscous models with $\alpha=1$ (middle model b) and top - model c)). These histograms show the percentage distribution of particles $N_{\text {count }}(\%)$ having a given number of neighbours $N_{\text {neigh. }}$. The average value $\left\langle N_{\text {neigh }}\right\rangle$, the final time $T$, as well as the injection velocity and from the inner Lagrangian point L1, are also reported.

The present conclusions do not disagree with our previous results, related to differently structured binary systems. Lanzafame \& Belvedere (2005) showed in a 2D SPH simulation that, even in the low compressibility regime, spirals and shock fronts do exist in accretion discs around supermassive black holes of $32 M_{\odot}$ or more, where a $\gamma=1.3$ and a stellar mass ratio $M_{1} / M_{2}>50$ were adopted. Similar results for 2D SPH modelling, but much less evident, were also shown in Lanzafame \& Belvedere (2001) and in Belvedere \& Lanzafame (2002). As explained in these three papers, the strong impact of the massive injected accreting stream (strongly accelerated in the deep primary potential well) with the outer disc edge 

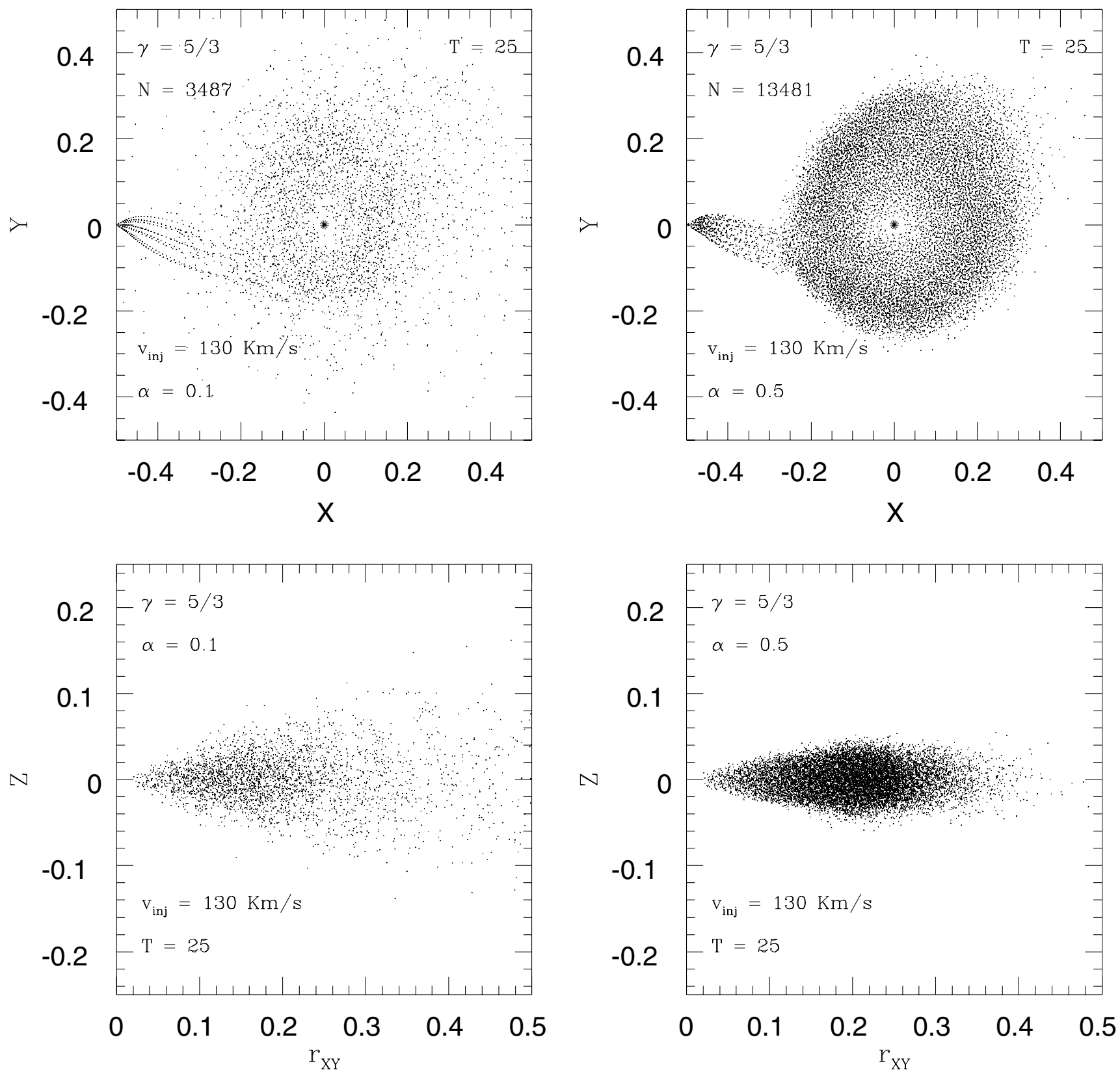

Fig. 6. $X Y$ and $r_{X Y} Z$ plots for the physically viscous models with $\alpha=0.1$ (left side) and with $\alpha=0.5$ (right side). The final time $T$ and total particle number $N$, as well as the injection velocity from the inner Lagrangian point $\mathrm{L} 1$, are also reported.

determines the onset of spiral shocks. Indeeed, for a massive primary star whose mass $M_{1}>8 \div 10 M_{\odot}$ and for a stellar mass ratio $M_{1} / M_{2}>10$, the gravitational acceleration of the massive primary dominates over both $-\nabla p / \rho$ and $(1 / \rho) \nabla \cdot \boldsymbol{t}$ terms.

A comparison of the absolute values of the pressure and viscosity forces per unit mass (here $(\nabla p / \rho$ and $(\nabla \cdot t) / \rho)$ is shown in Fig. 4 as a function of the radial distance for models $b$ ) and c) for all disc particles (with $\alpha=1$ ). These plots show that the work made by the pressure forces $W_{p}$ is of the same order as the viscous one $W_{v}$. This result suggests that physically viscous $\gamma=5 / 3$ low compressibility accretion disc models are meaningful if we adopt $\alpha \geq 0.1$.

\subsection{SPH accuracy}

A reasonable SPH accuracy is related to the number of space neighbours of each SPH particle. Problems about spatial resolution and SPH accuracy may exist for our physically inviscid accretion disc model. This model is a "reference model". Indeed, it is very hard to perform low compressibility gas dynamic simulations when attractive force fields are not dominant with respect to repulsive pressure forces. In the inviscid case, there is no way to obtain a particle concentration higher than 5-10 particle neighbours, even by changing the $h$ value in the range from its half to its double. Here, we do not want to challenge the SPH particle resolution required (commonly about 30-50 neighbours per particle in 3D simulations), but only to indicate that a problem exists in the astrophysical context of inviscid low compressibility modelling, when thermal energies are of the order of gravitational energies (generally of any binding energies) or larger. From a strictly mathematical point of view, the minimum condition of at least 3 non-coplanar neighbours SPH particles is statistically satisfied even in our "starting" inviscid disc model, 
following Ben Moussa \& Vila (2000). See the neighbours histograms in Fig. 5, showing the percentage distribution of particles $N_{\text {count }}(\%)$ having a given number of neighbours $N_{\text {neigh }}$ (and the average value $\left\langle N_{\text {neigh }}\right\rangle$ ) for all three a), b) and c) models.

However, our work is much more concerned with the introduction of physical viscosity. The main interest of this paper is the demonstration that, even in the case of very low compressibility, a strong physical viscosity allows the formation of a well bound accretion disc within the primary Roche Lobe. In some astrophysical situations, numerical simulations (e.g. Hernquist \& Katz 1989; Nelson \& Papaloizou 1994; Katz et al. 1996; Bate \& Burkert 1997; Spruit et al. 1987; Lanzafame \& Belvedere 2001) show a high particle concentration with 30 to 50 particle neighbours or more. According to the neighbours histograms shown in Fig. 7, we have an average number of 25.51 neighbours for the $\alpha=1$ high injection speed model (model b)) and 25.32 for the $\alpha=0.5$ high injection speed model, the latter being a further simulation carried out to investigate the role of $\alpha$, as shown below. These numbers seem to have a reasonable SPH accuracy.

\subsection{The role of viscosity}

Adopting $\alpha=1$ stresses differences in disc structure and dynamics with respect to the physically inviscid model. However, values of $\alpha$ less than unity may be more realistic according to some thin disc analytical models (Pringle et al. 1986; Lasota 2001). In order to examine the role of the physical viscosity strength, we carried out simulations with $\alpha=0.1$ and $\alpha=0.5$ with supersonic injection.

The mass transfer injection rates from L1 $\dot{M}_{\mathrm{inj}}$, accretion rates $\dot{M}_{\text {acc }}$ and ejection rates $\dot{M}_{\text {eje }}$ for the $\alpha=0.1$ and $\alpha=0.5$ models, are respectively $\dot{M}_{\text {inj }} \sim 9.6 \times 10^{16} \mathrm{~g} \mathrm{~s}^{-1}, \dot{M}_{\text {acc }} \sim 5.0 \times$ $10^{16} \mathrm{~g} \mathrm{~s}^{-1}$ and $\dot{M}_{\text {eje }} \sim 4.6 \times 10^{16} \mathrm{~g} \mathrm{~s}^{-1}$ and $\dot{M}_{\text {inj }} \sim 6.5 \times 10^{16} \mathrm{~g} \mathrm{~s}^{-1}$, $\dot{M}_{\text {acc }} \sim 6.5 \times 10^{16} \mathrm{~g} \mathrm{~s}^{-1}$ and $\dot{M}_{\text {eje }} \sim 6.5 \times 10^{13} \mathrm{~g} \mathrm{~s}^{-1}$.

Figure 6 shows $X Y$ and $r_{X Y} Z$ plots for these simulations, while Fig. 7 shows their neighbour histograms. For the $\alpha=$ 0.1 case no evident differences appear with respect to the inviscid reference model. A similar result was also reported in Lanzafame (2003). Instead, a well bound accretion disc is seen for the $\alpha=0.5$ case. These results show that in the low compressibility regime a value of $\alpha$ less or equal to 0.1 should not be effective. Low alpha values of the order of 0.1 are found in Pringle et al. (1986) and in Lasota (2001) in the thin disc approximation where hydrostatic equilibrium along the $\mathrm{Z}$ direction is assumed, a case different from ours, where turbulence plays a role also along the $Z$-axis. Therefore, we think that the $\alpha=0.5$ supersonic injection model is a good compromise and so can be considered as our reference viscid model. Statistically, the total number of particles is (slightly) larger than for the $\alpha=1$ model b). This result in our opinion is mainly due to a (slightly) lower particle radial transport toward the compact primary for the $\alpha=0.5$ (less viscosity) than for the $\alpha=1$ model b). The computed injection, accretion and ejection rates for these two models are in practice the same after $\sim 4$ orbital periods (stationary conditions). Here, no finer distinction can be made as the percentage computing errors are of the same order as the percentage difference in the number of particles.

Our physical viscosity is only a shear viscosity. For simplicity no bulk viscosity has been considered, as explicitly said in the paper. A value $\alpha=1$ for the bulk viscosity should be too high.

Figures 8 and 9 show in a logarithmic scale the angular momentum and temperature radial distribution respectively, for

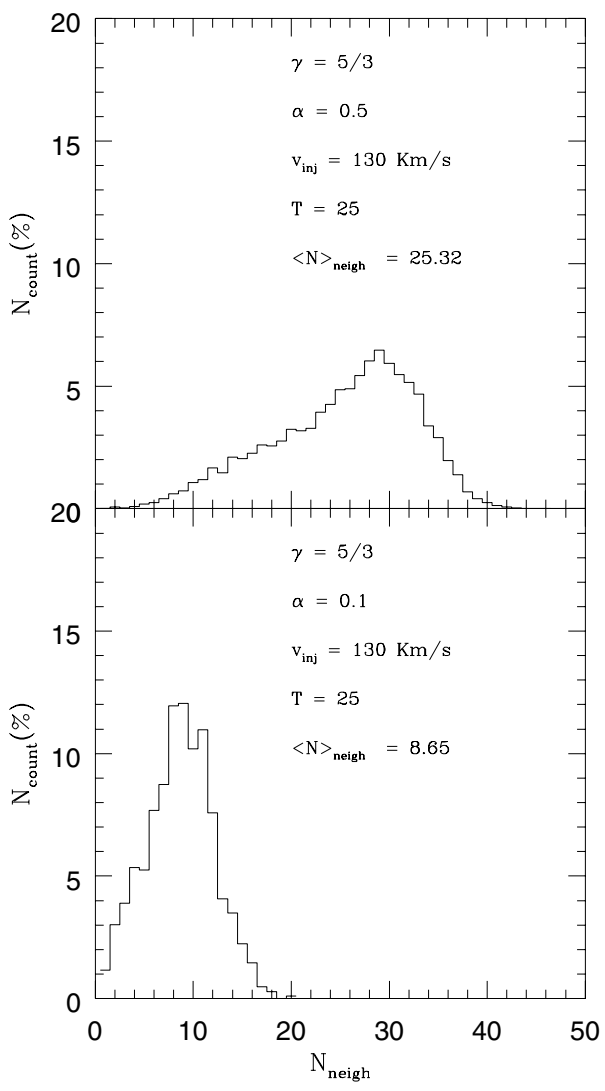

Fig. 7. Neighbours histograms for the physically viscous models with $\alpha=0.1$ (bottom) and with $\alpha=0.5$ (top). These histograms show the percentage distribution of particles $N_{\text {count }}(\%)$ having a given number of neighbours $N_{\text {neigh. }}$. The average value $\left\langle N_{\text {neigh }}\right\rangle$, the final time $T$, as well as the injection velocity from the inner Lagrangian point L1, are also reported.

models a), b) and c). The logarithmic angular momentum and temperature radial distributions, the radial profiles of pressure and viscosity forces, as well as the azimuthal profiles of the radial Mach number for $\alpha=0.5$ are similar to those for $\alpha=1$ model b) and, therefore, are not reported here.

The angular momentum radial distribution for the viscous models is very close to that of the standard model $J \propto r^{1 / 2}$. This can be explained considering that in stationary conditions an accretion disc redistributes the angular momentum injected at the outer edge into the disc bulk according to outer edge boundary conditions only, as already shown in Belvedere et al. (1993) and in Lanzafame et al. (1993). Physical viscosity plays a role in regions where particle velocity gradients are significant. This means that physical viscosity plays a relevant role mainly in the radial transport, while it has little influence on the tangential dynamics. A strong difference appears when looking at the temperature radial distribution. The heating effect of the physical viscosity is particularly evident in the disc inner zones. The disc itself is in an equilibrium stationary state in which the heated particles are directly accreted towards the primary. This as for particle advection. As for conduction, although much less important, the temperature decreases towards the exterior, thus heat is dispersed outside. However, discs could also radiate energy. In disc models without explicit inclusion of radiative terms in the energy equation (almost all models, since, such an inclusion complicates the analysis), the effect of radiative cooling is better simulated with $\gamma$ less than $5 / 3$. Thus, we cannot neglect the possibility that the non-appearance of shocks in viscid discs may be 


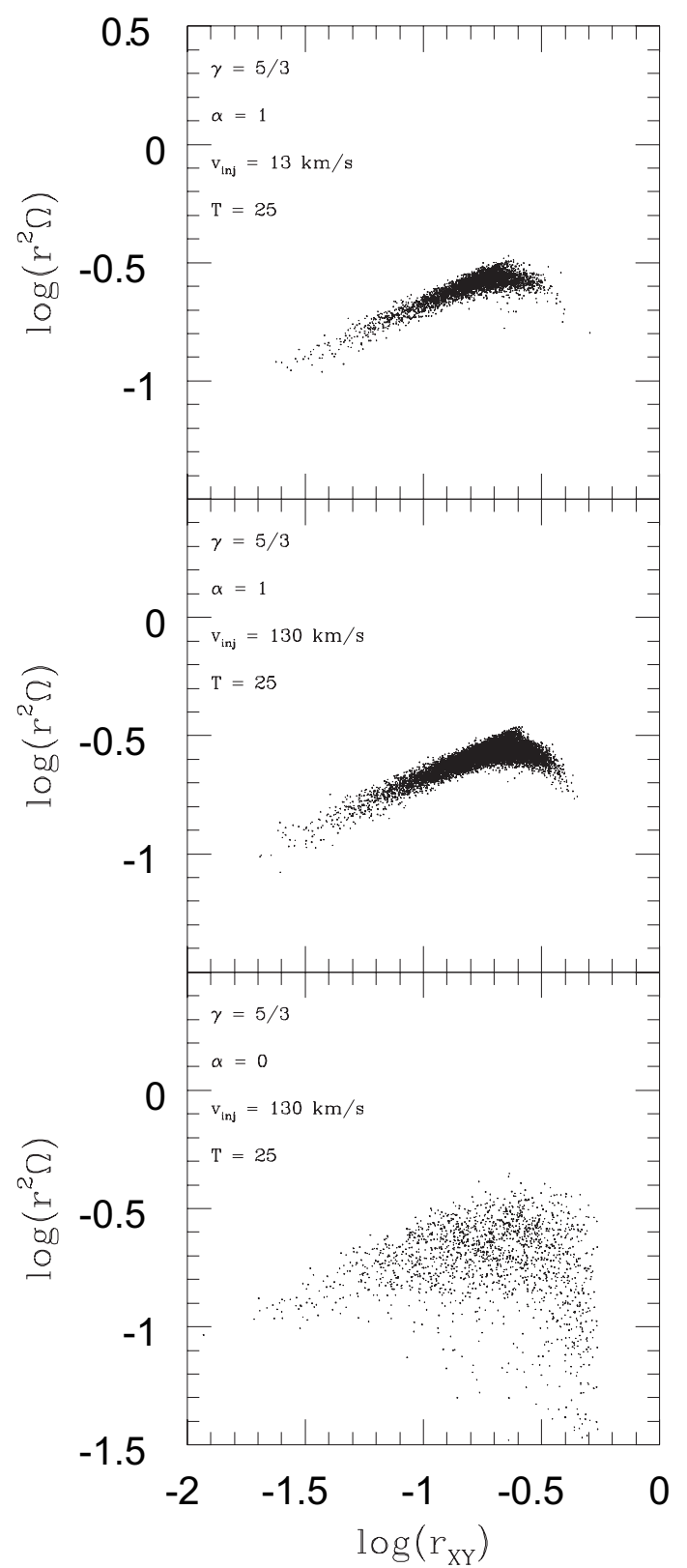

Fig. 8. Logarithmic plots of angular momentum radial distributions for the inviscid model a) with $\alpha=0$ (bottom) and the two physically viscous models with $\alpha=1$ (middle - model b) and top - model c)). The final time $T$ as well as the injection velocity from the inner Lagrangian point $\mathrm{L} 1$, are also reported.

a consequence of the lack of an efficient radiative cooling in the simulations.

\subsection{Concluding remarks}

The main consequences of the viscous heating, which implies a more efficient particle radial transport toward the primary, in the case of low compressibility accretion disc modelling, are the following: disc bounding inside the primary Roche lobe, disc thinning as far as their latitudinal spread is concerned, disc heating and its increasing in temperature radial gradient. Therefore, in viscous discs particles are hotter and much better accreted (see the comparison between the values of the accretion rate for models b) and c)). Indeed, for the first time, our results show that

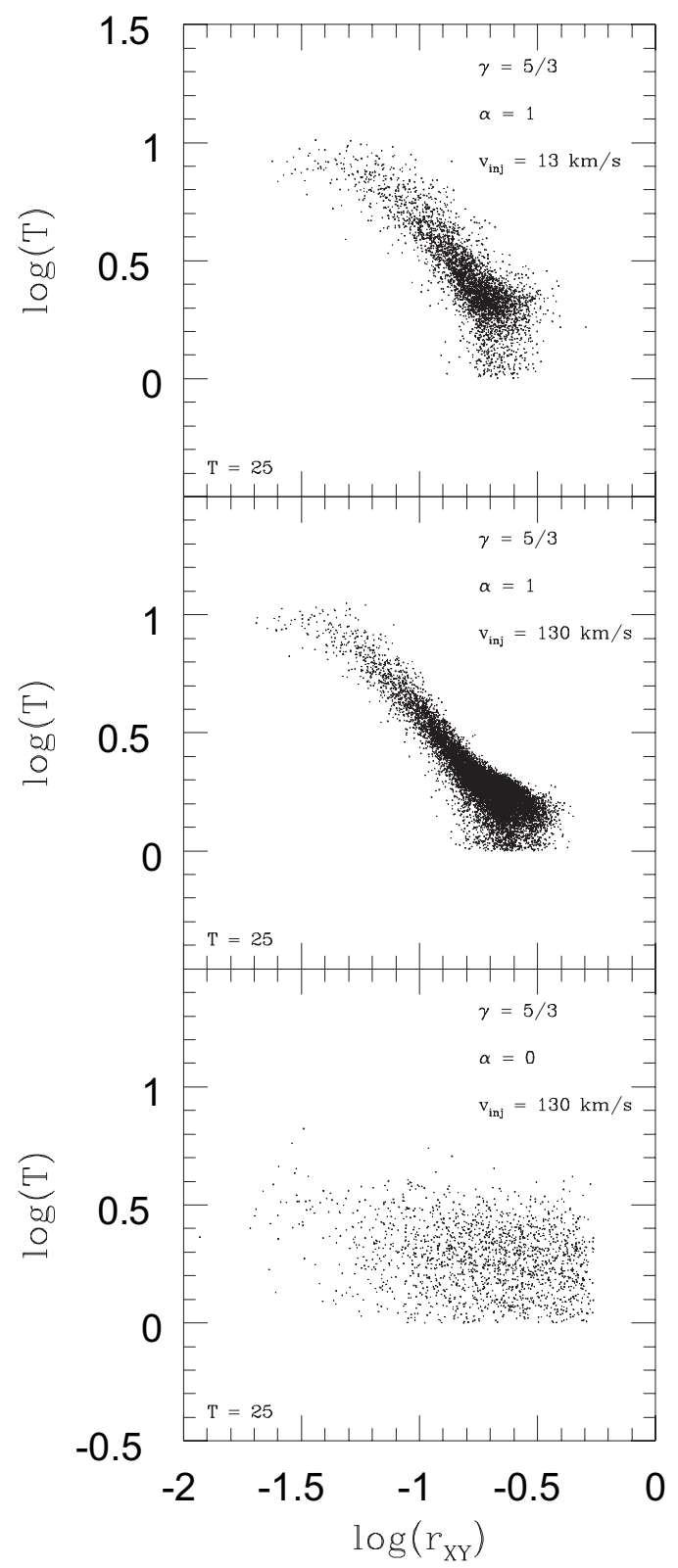

Fig. 9. Logarithmic plots of temperature radial distributions for the inviscid model a) with $\alpha=0$ (bottom) and the two physically viscous models with $\alpha=1$ (middle - model b) and top - model c)). The final time $T$ as well as the injection velocity from the inner Lagrangian point $\mathrm{L} 1$, are also reported.

physical viscosity supports the development of a well bound accretion disc in the primary potential well, even in the case of low compressibility. However, no spirals and shocks appear, although the disc structure is well defined and statistically meaningful, but this may be a consequence of the lack of an efficient radiative cooling, as said before. Such results mean that the initial angular momentum injection conditions at the disc outer edge are responsible for the disc tangential dynamics, while viscosity is mainly responsible for the thermodynamical disc properties, even for low compressibility disc models $(\gamma>1.1$, here $\gamma=5 / 3$ ). Indeed, physical viscosity implies a degradation and smoothing effect both on dynamical and thermodynamical discontinuities, so disfavouring shocks. As shown in Lanzafame (2003), high compressibility $(\gamma \leq 1.01)$ is a quite favourable 
condition to develop spirals and shock fronts in the radial flow according to some suitable dynamical and thermodynamical initial outer edge conditions at L1. Accretion disc models around massive black holes, as in Lanzafame \& Belvedere (2005), are an exception. For such objects, spirals and shock fronts develop anyway due to the strong impact of the flow injected from L1. In this case both dynamical and thermodynamical disc properties are mainly a consequence of the primary strong gravitational field.

Acknowledgements. We are grateful to the anonymous referee for helpful comments and suggestions.

\section{References}

Bate, M. R., \& Burkert, A. 1997, MNRAS, 288, 1060

Belvedere, G., Lanzafame, G., \& Molteni, D. 1993, A\&A, 280, 525

Belvedere, G., \& Lanzafame, G. 2002, PASJ, 54, 781

Ben Moussa, B., \& Vila, J. P. 2000, SIAM, J. Numer. Anal., 37, 863

Bisikalo, D. V., Boyarchuk, A. A., Chechetkin, V. M., Kutznetsov, O. A., \& Molteni, D. 1999, Astro. Rep., 43, 797

Bisikalo, D. V., Boyarchuk, A. A., Kutznetsov, O. A., \& Chechetkin, V. M. 2000, Astronomy Rep., 44, 26

Blondin, J. M. 2000, New Astron., 5, 53

Boris, J. P., \& Book, D. L. 1973, J. Comput. Phys., 12, 1198

Chakrabarti, S. K. 1992, MNRAS, 259, 410

Drimmel, R. 1993, MNRAS, 282, 982

Flebbe, O., Münzel, H., Riffert, H., \& Herold, H. 1992, Mem. Soc. Astr. It., 65, 1049

Flebbe, O., Münzel, H., Herold, H., Riffert, H., \& Ruder, H. 1994, ApJ, 431, 754

Hernquist, L., \& Katz, N. 1989, ApS, 70, 419

Ichikawa, S., \& Osaki, Y. 1992, PASJ, 44, 15

Ichikawa, S., \& Osaki, Y. 1994, PASJ, 46, 621

Kaisig, M. 1989, 2003, A\&A, 218, 102

Katz, N., Weinberg, D. H., \& Hernquist, L. 1996, ApJS, 105, 19
Landau, L. D., \& Lifshitz, E. M. 1959, Course of Theoretical Physics, Vol. VI: Fluid Mechanics (Pergamon Press)

Lanzafame, G. 2003, A\&A, 403, 593

Lanzafame, G., \& Belvedere, G. 1997, MNRAS, 284, 957

Lanzafame, G., \& Belvedere, G. 1998, MNRAS, 295, 618

Lanzafame, G., \& Belvedere, G. 2001, JKAS, 34, S313

Lanzafame, G., \& Belvedere, G. 2005, ApJ, 632, 499

Lanzafame, G., Belvedere, G., \& Molteni, D. 1992, MNRAS, 258, 152

Lanzafame, G., Belvedere, G., \& Molteni, D. 1993, MNRAS, 263, 839

Lanzafame, G., Maravigna, F., \& Belvedere, G. 2000, PASJ, 52, 515

Lanzafame, G., Maravigna, F., \& Belvedere, G. 2001, PASJ, 53, 139

Lasota, J. P. 2001, New Astr. Rev., 45, 449

Lattanzio, J. C., Monaghan, J. J., Pongracic, H., \& Schwarz, M. P. 1986, SIAM

J. Sci. Stat. Comput., 7, 591

Makita, M., Miyawaki, K., \& Matsuda, T. 2000, MNRAS, 316, 906

Matsuda, T., Sekino, N., Shima, E., Sawada, K., \& Spruit, H. 1990, A\&A, 235, 211

Matsuda, T., Ishii, T., Sekino, N., et al. 1992, MNRAS, 255, 183

Meglicki, Z., Wickramasinghe, D., \& Bicknell, G. V. 1993, MNRAS, 264, 691

Molteni, D., Belvedere, G., \& Lanzafame, G. 1991, MNRAS, 249, 748

Monaghan, J. J. 1985, Comp. Phys. Rep., 3, 71

Monaghan, J. J. 1992, ARA\&A, 30, 543

Monaghan, J. J., \& Lattanzio, J. C. 1985, A\&A, 149, 135

Murray, J. R. 1996, MNRAS, 279, 402

Nelson, R. 1994, Mem. S.A. It, 65, 1161

Nelson, R. P., \& Papaloizou, J. C. B. 1994, MNRAS, 270, 1

Pringle, J. E., Verbunt, F., \& Wade, R. A. 1986, MNRAS, 221, 169

Savonije, G. J., Papaloizou, J. C. B., \& Lin, D. N. C. 1994, MNRAS, 268, 13

Sawada, K., Matsuda, T., Inoue, M., \& Hachisu, I. 1987, MNRAS, 224, 307

Sawada, K., \& Matsuda, T. 1992, MNRAS, 255, 17p

Shakura, N. I. 1972, Astron. Zh., 49, 921 (English tr.: 1973, Sov. Astron., 16, 756)

Shakura, N. I., \& Sunyaev, R. A. 1973, A\&A, 24, 337

Springel, V., Yoshida, N., \& White, S. D. M. 2001, New Astr., 6, 79

Spruit, H. C., Matsuda, T., Inoue, M., \& Sawada, K. 1987, MNRAS, 229, 517

Truss, M. R., Murray, J. R., Wynn, G. A., \& Edgar, R. G. 2000, MNRAS, 319, 467

Truss, M. R., Murray, J. R., \& Wynn, G. A. 2001, MNRAS, 324, L1

Yukawa, H., Boffin, H. M. J., Matsuda, T., \& Inoue, M. 1997, MNRAS, 292, 321 